\title{
Einfluss von Klima und Baumvitalität auf den Be- fall von Waldföhren durch rindenbrütende Insekten
}

\author{
Beat Wermelinger \\ Martin M. Gossner \\ Doris Schneider Mathis \\ Dieter Trummer \\ Andreas Rigling
}

\begin{abstract}
Eidgenössische Forschungsanstalt für Wald, Schnee und Landschaft $(\mathrm{CH})^{*}$ Eidgenössische Forschungsanstalt für Wald, Schnee und Landschaft $(\mathrm{CH})$ Eidgenössische Forschungsanstalt für Wald, Schnee und Landschaft $(\mathrm{CH})$ Eidgenössische Forschungsanstalt für Wald, Schnee und Landschaft $(\mathrm{CH})$ Eidgenössische Forschungsanstalt für Wald, Schnee und Landschaft $(\mathrm{CH})$
\end{abstract}

\section{Einfluss von Klima und Baumvitalität auf den Befall von Waldföhren durch rindenbrütende Insekten}

Die Entwicklung von Insekten hängt zwar in erster Linie von der Temperatur ab, wichtig sind jedoch auch die Inhaltsstoffe ihrer Wirtspflanzen. Einerseits ist deren Nahrungsqualität, insbesondere der Stickstoffgehalt, für die Insektenvermehrung sehr wichtig, andererseits beeinflussen toxische Abwehrstoffe der Pflanzen die Besiedlung und Entwicklung von Insekten. Die Produktion dieser Abwehrstoffe ist bei Trockenheit vielfach stark eingeschränkt. Im Wallis wurden in den vergangenen 15 Jahren verschiedene Untersuchungen zum Einfluss von Temperatur und Trockenheit auf rindenbrütende Insekten durchgeführt. Die aggressivsten Arten, das heisst, diejenigen mit dem grössten Befallspotenzial, waren der Sechszähnige Föhrenborkenkäfer (Ips acuminatus), der Blaue Föhrenprachtkäfer (Phaenops cyanea) und - in geringerem Mass - der Kleine Waldgärtner (Tomicus minor). Sie waren in der Lage, Waldföhren auch bei relativ hoher Vitalität zu besiedeln. Generell stieg der Befall durch diese Arten sowie andere rindenbrütende Käfer mit zunehmender Kronenverlichtung an. Die räuberischen Insekten zeigten dasselbe Besiedlungsmuster. Die Intensität des Reifungsfrasses des Waldgärtners hing von der Kronenverlichtung ab, wurde aber signifikant von der Wasserversorgung der Bäume beeinflusst. Die zeitliche Entwicklung der Befallsdichten aggressiver Rindenbrüter folgte in den untersuchten Jahren ziemlich genau einem Index für Trockenstress der Wirtsbäume. Die Befallsraten zeigten allerdings oft deutliche regionale Unterschiede. Die Brutdichten nahmen mit der Höhe über Meer zu, was auf die abnehmende Zahl ihrer natürlichen Feinde und auf den bei tieferen Temperaturen reduzierten Harzfluss zurückzuführen sein dürfte.

Keywords: tree decline, insect pests, resistance, vitality, drought, defoliation doi: $10.3188 /$ szf.2018.0251

*Zürcherstrasse 111, CH-8903 Birmensdorf, E-Mail beat.wermelinger@wsl.ch

$\mathrm{I}$ n den letzten Jahrzehnten haben die Jahresdurchschnittstemperaturen und die Häufigkeit von Trockenperioden weltweit zugenommen, was bereits in verschiedenen Wäldern auf allen Kontinenten zu erhöhter Baummortalität geführt hat (Allen et al 2010). Diese Mortalität kann physiologisch bedingt sein, aber auch durch abiotische (Feuer, Wind) und biotische (Insekten, Pathogene) Faktoren oder eine Kombination derselben verursacht werden. Unter den Insekten sind speziell Borkenkäferarten dafür bekannt, dass sie geschwächte Bäume besiedeln und zum Absterben bringen können, was im ökologischen Zusammenhang eine positive Selektion der vitalen Baumindividuen und das Schaffen neuer Lebensräume bedeutet (Müller et al 2008, Wermelinger 2017). Allerdings können einige Borkenkäfer- und andere Insektenarten unter gewis- sen Voraussetzungen zu grossflächigem Massenbefall insbesondere von Nadelbäumen führen und damit die von uns erwarteten Waldleistungen wie Holz, Schutz oder Erholung vorübergehend empfindlich schmälern - sie werden zu Schädlingen. Borkenkäfer werden generell als der wichtigste einzelne Mortalitätsfaktor betrachtet (Das et al 2016).

Die Entwicklung von Insektenpopulationen steht in direktem Zusammenhang mit den herrschenden Witterungsbedingungen. Insbesondere bewirken höhere Temperaturen, dass gewisse Arten zusätzliche Generationen pro Jahr entwickeln und damit mehr Nachkommen produzieren (Wermelinger \& Seifert 1999).

Die Witterungsbedingungen verändern aber auch die Attraktivität eines Wirtsbaumes gegenüber möglichen Schadorganismen. Neben dem quantita- 


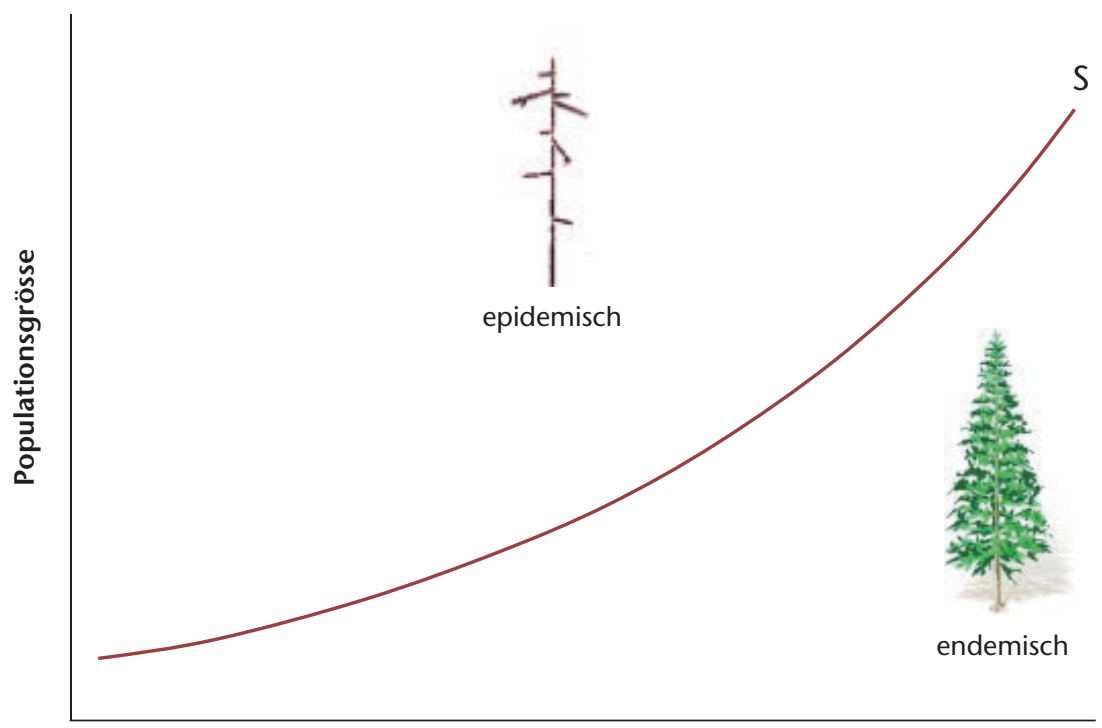

Baumvitalität

Abb 1 Abhängigkeit der für einen Befall notwendigen Mindestpopulationsgrösse (Schwellenwert S) von der Baumvitalität. Je vitaler ein Baum ist, desto grösser muss die Käferpopulation sein, um ihn besiedeln zu können. Quelle: Wermelinger \& Jakoby (im Druck)

tiven Angebot von Wirtsbäumen spielt deshalb auch die Zusammensetzung ihrer Inhaltsstoffe bei der Besiedlung, Eiablage und Entwicklung der Insekten eine äusserst wichtige Rolle. Toxische Abwehrstoffe wie Phenole, Terpene oder Alkaloide beeinträchtigen die Entwicklung und erhöhen die Mortalität der Herbivoren (Pflanzenfresser) unter ihnen (Larsson 2002, Phillips \& Croteau 1999). Insbesondere Trockenheit kann die Produktion solcher Abwehrstoffe und damit die Resistenz der Bäume herabsetzen und zu stärkerem Befall durch Borkenkäfer führen (Gaylord et al 2013, Netherer et al 2015).

Der vorliegende Artikel gibt einen Überblick über die generelle Bedeutung der Baumdisposition für einen Befall durch herbivore Insekten und fasst anschliessend Erkenntnisse aus gut 15 Jahren Untersuchungen im Wallis zu den Interaktionen zwischen
Waldföhren (Pinus sylvestris L.) und rindenbrütenden Insekten zusammen.

\section{Bedeutung der Baumvitalität für Insekten}

Als Resistenz gegen Herbivore wird die Fähigkeit von Pflanzen bezeichnet, die Vermehrung von pflanzenfressenden Insekten herabzusetzen (Karban \& Baldwin 1997). Ein einfach zu erhebendes Mass für diese Fähigkeit zu finden, ist schwierig, denn die Resistenz und damit auch die Vitalität eines Baumes können nicht mit einem einfachen Parameter beschrieben werden. Die im Rahmen der Waldsterbensdebatte entstandenen «Waldschadeninventuren» verwenden als Indikator für den Gesundheitszustand eines Baumes seine Kronenverlichtung. Allerdings hängt diese auch von anderen Faktoren wie Standortbedingungen, Klima, Witterung sowie von der momentanen Aktivität von herbivoren Insekten oder Pathogenen ab. Im Folgenden wird - mangels besserer Alternativen - die Kronenverlichtung eines Baumes als Mass für die Reduktion seiner Vitalität bzw. Resistenz verwendet.

Die Resistenz eines lebenden Baumes gegen Borkenkäfer und andere Rindenbesiedler hängt stark von den aktuellen oder den kurz zuvor herrschenden Witterungsbedingungen ab (z.B. Raffa et al 2008). Heisse Sommer, die den Wasserbedarf der Bäume erhöhen (Williams et al 2013), sind meist gepaart mit geringen Niederschlägen, was zusammen die Wasserverfügbarkeit einschränkt und den Trockenstress verstärkt. Dadurch können weniger Abwehrstoffe wie die aus Terpenen bestehenden Baumharze produziert werden (Netherer et al 2015). Einem Einbohrversuch eines Borkenkäfers begegnet ein Nadelbaum durch Freisetzen von zuvor eingelagertem Harz. Dies erlaubt es ihm, schnell auf Käferbefall zu reagieren und einzeln einbohrende Tiere abzuhal-
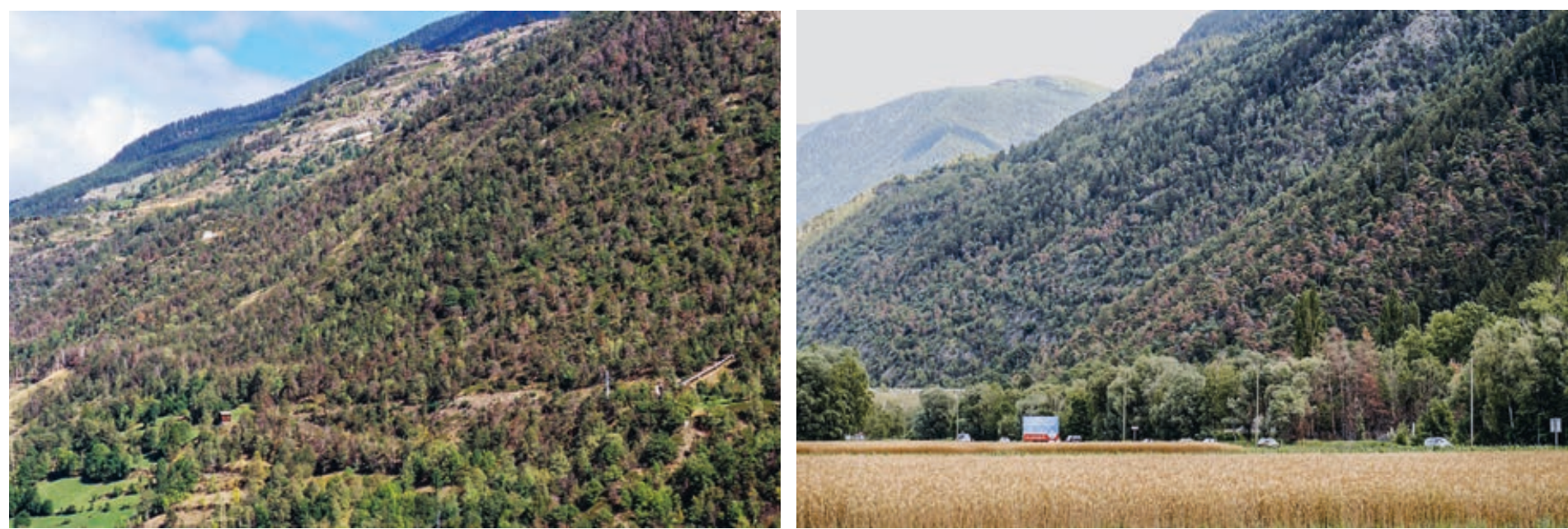

Abb 2 Ausgedehnte Föhrenmortalität im Wallis Ende der 1990er-Jahre im Vispertal (Stalden, Oktober 2000; links) und 2016/2017 im Rhonetal (Visp, Juni 2017; rechts). Fotos: Beat Wermelinger 
ten, einzuschliessen oder zu vergiften. Können sich trotzdem Käfer einbohren, induziert dies eine Neusynthese toxischer Harzkomponenten, die das Etablieren der Käfer und ihrer Brut verhindern sollen (Krokene 2015).

Für die Besiedlung eines lebenden Baumes ist deshalb eine Mindestzahl von einbohrenden Käfern nötig, um die Baumabwehr überwinden zu können. Dieser Schwellenwert ist abhängig von der Abwehrfähigkeit der Wirtsbäume (Abbildung 1). Je stärker ein Wirtsbaum unter (Trocken-)Stress steht, desto kleiner ist die für einen Befall notwendige Populationsdichte.
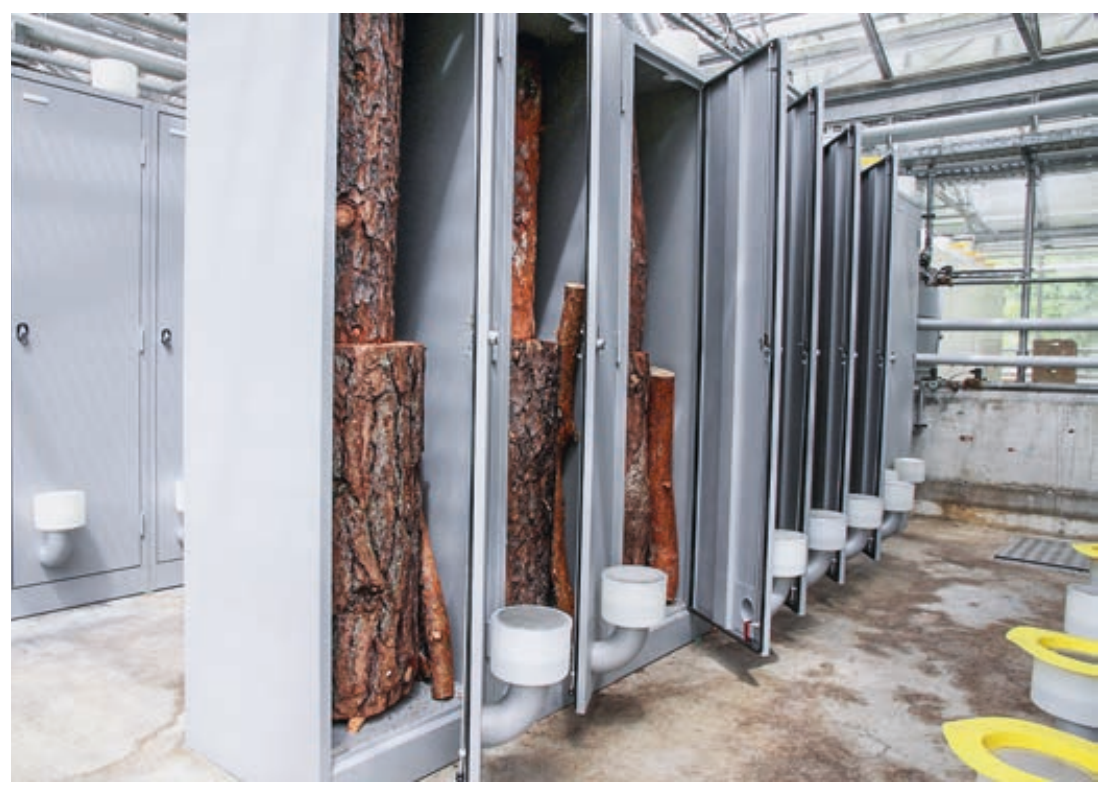

Abb 3 In sogenannten Fotoeklektoren wurden Stamm- und Aststücke zum Ausbrüten von Insekten inkubiert. In die geschlossenen Schränke fällt Licht nur über die unten und oben angebrachten Fangdosen. Die ausgeschlüpften Insekten streben zum Licht und können in den Dosen abgesammelt werden. Foto: Beat Wermelinger
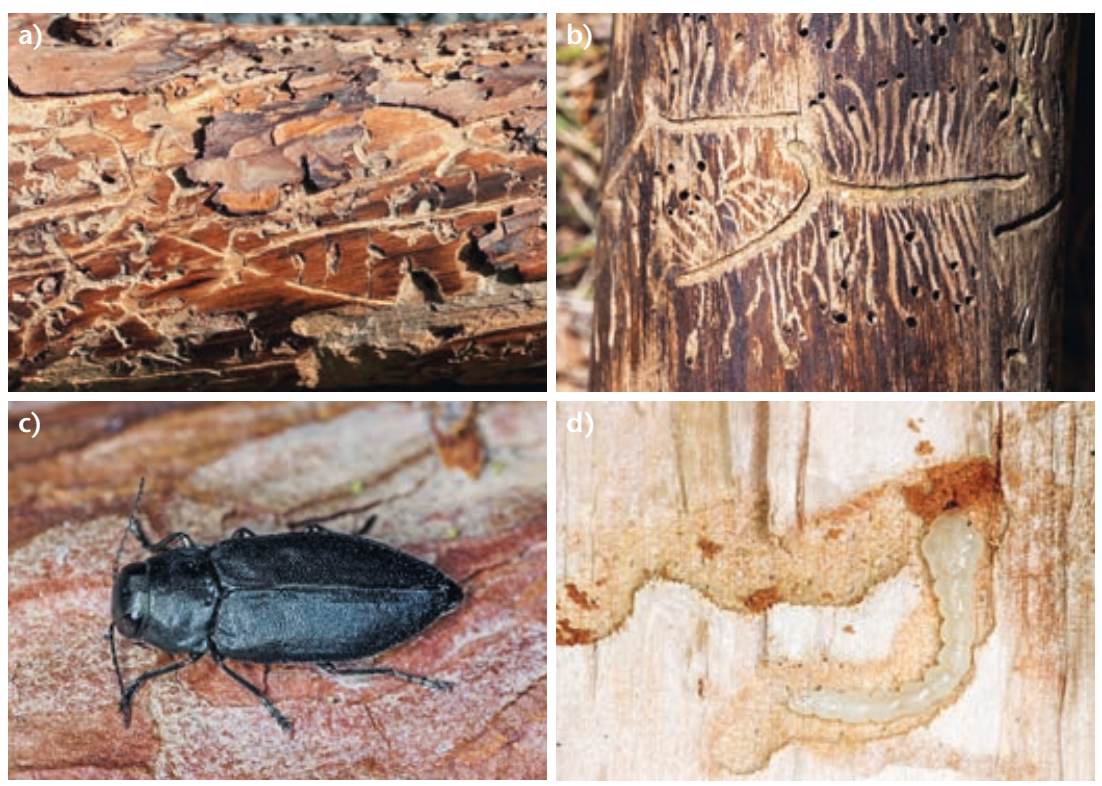

Abb 4 Die Käferarten mit dem grössten Befallspotenzial vitaler Waldföhren: a) Sechszähniger Föhrenborkenkäfer (Ips acuminatus; Brutbild), b) Kleiner Waldgärtner (Tomicus minor; Brutbild), c, d) Blauer Föhrenprachtkäfer (Phaenops cyanea; Adulttier sowie Larve und Frassgang). Fotos: Beat Wermelinger

\section{Kronenverlichtung und Käferbefall im Wallis}

In den 1990er- und frühen 2000er-Jahren fielen die Waldföhren in verschiedenen Gebieten des Wallis, in weiteren inneralpinen Tälern der Schweiz sowie in angrenzenden Ländern durch eine ausgeprägte Mortalität auf - ein Phänomen, das seit 2016/2017 in der Region Visp/Brig erneut auftritt (Abbildung 2). Es zeigte sich, dass Trockenheit der wichtigste auslösende Faktor war, vor allem als Folge von zunehmend hohen Sommertemperaturen (Rebetez \& Dobbertin 2004). Speziell die Frühjahrstrockenheit schwächte die Bäume und verbesserte die Befallsbedingungen für die rindenbrütenden Insekten.

\section{Föhrensterben der 2000er-Jahre}

In einer Untersuchung in Salgesch und Stalden wurden von 2000 bis 2004 jedes Jahr Waldföhren unterschiedlicher Verlichtungsgrade gefällt. Aus jedem der insgesamt 209 Bäume wurden zwei Stammabschnitte von je $75 \mathrm{~cm}$ Länge (in Brusthöhe und beim Kronenansatz) sowie zwei Aststücke von je $100 \mathrm{~cm}$ Länge herausgeschnitten. An der Eidgenössischen Forschungsanstalt für Wald, Schnee und Landschaft (WSL) wurden die Stücke inkubiert, um die sich darin entwickelnden Insekten ausschlüpfen zu lassen (Abbildung 3; detaillierte Methodik siehe Wermelinger et al 2008).

Von den gefundenen 41 holz- und rindenfressenden Insektenarten wurden 3 als mässig oder stark aggressiv bezeichnet, da sie auch vitalere Bäume mit nur geringer Kronenverlichtung befielen (Wermelinger et al 2008): die beiden Borkenkäferarten Ips acuminatus (Sechszähniger Föhrenborkenkäfer) und Tomicus minor (Kleiner Waldgärtner) sowie der Prachtkäfer Phaenops cyanea (Blauer Föhrenprachtkäfer) (Abbildung 4). Andere Rindenbrüter unter den Rüssel-, Pracht- und Bockkäfern befielen nur stärker verlichtete Bäume von geringerer Vitalität und erreichten deutlich geringere Dichten.

Es zeigte sich generell eine deutliche Abhängigkeit der Dichte rindenbrütender Borken- und anderer Rüsselkäfer sowie der Prachtkäfer vom Verlichtungsgrad ihrer Wirtsbäume zum Zeitpunkt der Besiedlung (Abbildung 5). Bäume mit hoher (65-80\%) und sehr hoher (85-100\%) Kronenverlichtung und damit geringer Vitalität waren meist signifikant stärker befallen als solche mit geringerer Verlichtung. Allerdings blieb auch von den Bäumen mit sehr hoher Kronenverlichtung mehr als die Hälfte von diesen Rindenbrütern unbesiedelt. Aus 20\% dieser Bäume schlüpften überhaupt keine Insekten. Der Sechszähnige Föhrenborkenkäfer und der Blaue Föhrenprachtkäfer waren hingegen auch in relativ vitalen Bäumen mit einer Verlichtung von nur 30\% (Wermelinger et al 2008) zu finden, was ihre Aggres- 


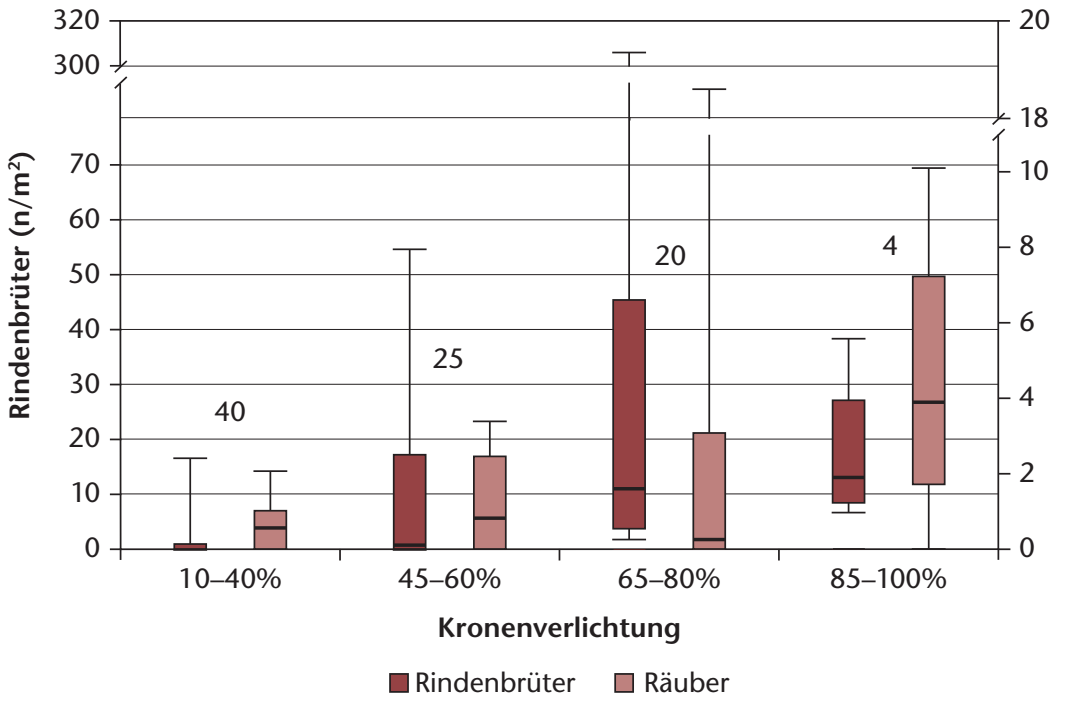

Abb 5 Besiedlungsdichte rindenbrütender Rüssel- (Curculionidae, inkl. Borkenkäfer) und Prachtkäfer (Buprestidae) sowie räuberischer Insekten (Käfer, Fliegen, Kamelhalsfliegen) in Waldföhren mit unterschiedlicher Kronenverlichtung zum Zeitpunkt der Eiablage (Jahre 2000-2004). Die Zahlen oberhalb der Boxen bezeichnen die Anzahl Bäume mit Befall durch die genannten Artengruppen. Mit generalisierten linearen gemischten Modellen (Poisson-GLMM) und anschliessenden Wald-Chi2-Tests wurde auf Unterschiede zwischen den Kronenverlichtungsklassen getestet (Rindenbrüter: Chi $^{2}=1426.6, p<0.001$; Räuber: $\left.\mathrm{Chi}^{2}=68.508, p<0.001\right)$.

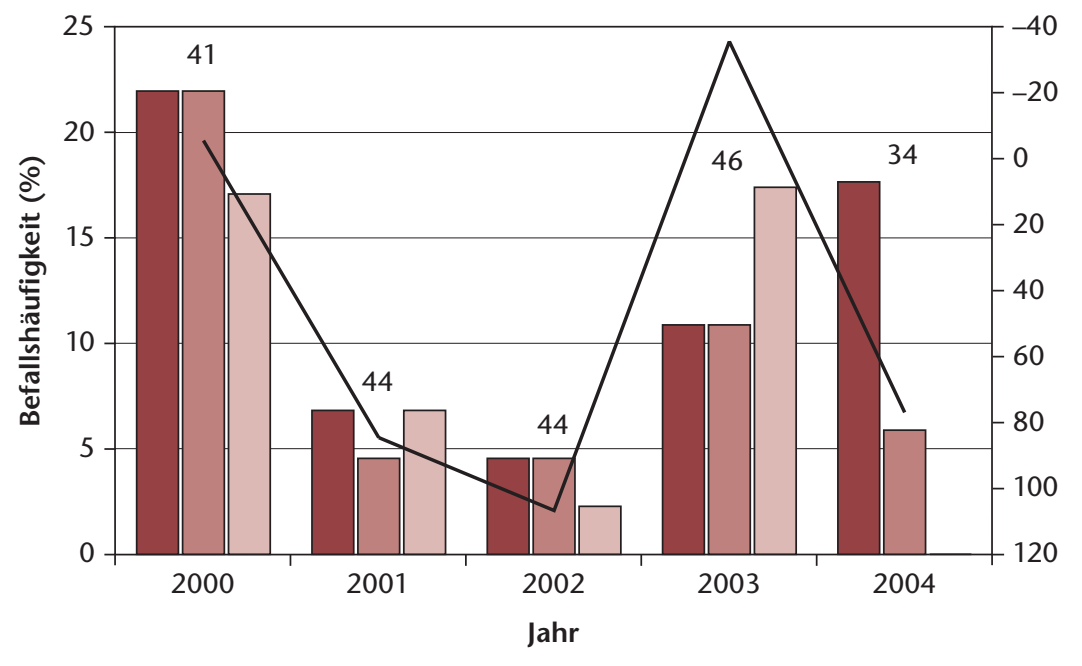

Tomicus minor $\quad \square$ Phaenops cyanea $\quad \square$ Pissodes piniphilus - Trockenheitsindex

Abb 6 Befallshäufigkeit von Waldföhren durch den Kleinen Waldgärtner (Tomicus minor), den Blauen Föhrenprachtkäfer (Phaenops cyanea) und den Föhrenstangenrüssler (Pissodes piniphilus) an den beiden Standorten Salgesch und Stalden zwischen 2000 und 2004 sowie Trockenheitsindex (Niederschlag minus potenzielle Evapotranspiration = klimatische Wasserbilanz). Die Zahlen oberhalb der Säulen bezeichnen die Anzahl untersuchter Bäume. Die Unterschiede im Befall zwischen den Jahren waren nur für den Föhrenstangenrüssler signifikant (binomiales Regressionsmodell GLMM mit Wald-Chi-Test; Kleiner Waldgärtner: $\mathrm{Chi}^{2}=6.179, p=0.186$; Blauer Föhrenprachtkäfer: $\mathrm{Chi}^{2}=7.496, p=0.112$; Föhrenstangenrüssler: Chi $^{2}=9.497, p=0.049$ ).

sivität zeigt. Andere als schädlich bekannte Käferarten wie der Kleine Waldgärtner oder der Föhrenstangenrüssler (Pissodes piniphilus) waren in stark verlichteten Bäumen mit rund 70-80\% Kronenverlichtung am zahlreichsten (Wermelinger et al 2008). Die Befallsdichten des Sechszähnigen Föhrenborkenkäfers und des Blauen Föhrenprachtkäfers waren von der Veränderung der Kronenverlichtung im vergangenen Jahr unbeeinflusst (Wermelinger et al 2008). Die aggressiven, also vitale Bäume befallenden Käferarten besiedelten vor allem 100- bis 130-jährige Bäume. Allerdings wies auch in dieser Alterskategorie nur rund ein Drittel der Bäume einen Befall durch diese Arten auf.

Die Dichte der räuberischen Käfer, Fliegen und Kamelhalsfliegen war in der höchsten Verlichtungsklasse am grössten (Abbildung 5). Dies bedeutet, dass auch die Besiedlungsdichte der Rindenbrüter in extrem geschwächten Bäumen am höchsten gewesen sein dürfte, der Schlupferfolg der Rindenbrüter aber durch die Wirkung natürlicher Feinde begrenzt wurde. Es ist zu beachten, dass in dieser Untersuchung keine parasitischen Wespen berücksichtigt wurden.

Neben den Stämmen wurden auch Wurzelstücke (313 Stück, total 159 m Länge) von 64 Waldföhren inkubiert, um auch wurzelbrütende Käfer zu beurteilen. So können Borkenkäfer der Gattung Hylastes die Baumverjüngung negativ beeinflussen. Sie entwickeln sich in Wurzelstöcken, die adulten Käfer vollziehen danach ihren Reifungsfrass an den Stämmchen junger Bäume. Diese Käfer schlüpften fast ausschliesslich (96\%) aus Bäumen mit mindestens $85 \%$ Verlichtung. Sie bevorzugen somit Wurzeln absterbender Bäume als Brutsubstrat.

Aus faunistischer bzw. naturschützerischer Sicht interessant waren die Funde des Jagdkäfers Temnochila caerulea, der in Mitteleuropa als Urwaldreliktart gilt (Eckelt et al 2017). Dieser Räuber entwickelte sich erstaunlicherweise überwiegend in wenig verlichteten Föhren und erbeutete dort vor allem phytosanitär irrelevante Arten wie den Weichen Nagekäfer (Ernobius mollis). Dieser lebt in der äusseren, toten Borke und nicht im lebenden Bast wie viele Borkenkäfer und hat deshalb für die Vitalität der Bäume keine Bedeutung. Er war in Bäumen jeglichen Verlichtungsgrades präsent und in den vitalsten Bäumen sogar am häufigsten (Wermelinger et al 2008). Bäume mit sehr starker Verlichtung wurden recht zahlreich vom Zimmermannsbock (Acanthocinus aedilis) besiedelt (Wermelinger et al 2008), der in der Schweiz auf der Roten Liste steht (Monnerat et al 2016).

Der Einfluss der Trockenheit auf den Käferbefall zeigte sich während der fünfjährigen Untersuchung deutlich (Abbildung 6). Der Trockenheitsindex (klimatische Wasserbilanz $=$ Niederschlag minus potenzielle Evapotranspiration) der Periode Januar-Mai war in zwei der fünf Jahre deutlich höher, vor allem im berühmten Hitze- und Trockenjahr 2003. Fast parallel dazu verlief der Befall durch den Kleinen Waldgärtner, den Blauen Föhrenprachtkäfer und den Föhrenstangenrüssler. Allerdings blieb im Jahr 2004 die Besiedlungsrate des Kleinen Waldgärtners aus unbekannten Gründen hoch. 

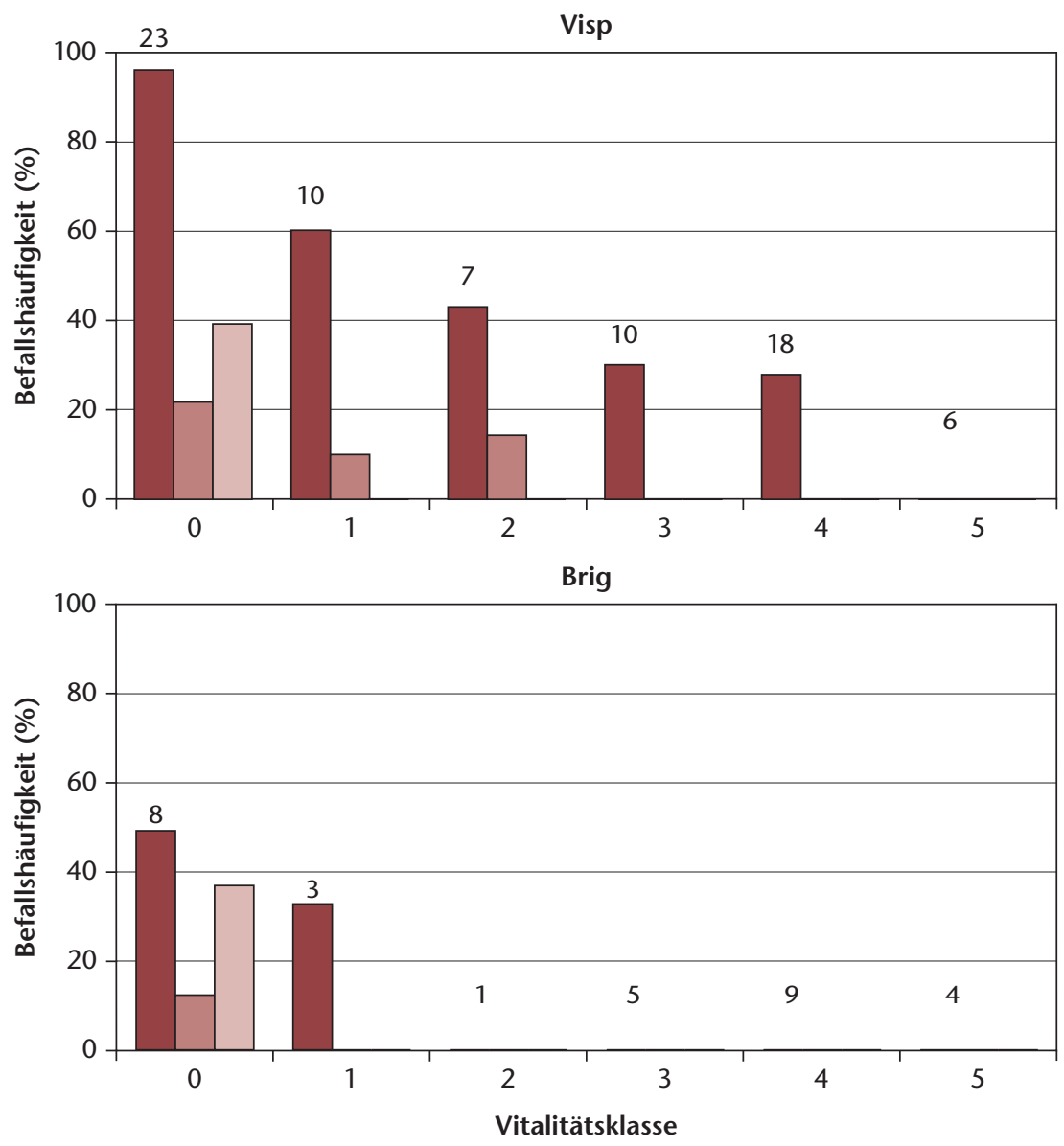

$\square$ Phaenops cyanea $\square$ Ips acuminatus $\square$ Tomicus minor

Abb 7 Befallshäufigkeit von Waldföhren unterschiedlicher Vitalität $(0=$ tot bis $5=$ vital, Klassierung s. Text) durch den Blauen Föhrenprachtkäfer (Phaenops cyanea), den Sechszähnigen Föhrenborkenkäfer (Ips acuminatus) und den Kleinen Waldgärtner (Tomicus minor) im Jahr 2017. Die Zahlen oberhalb der Vitalitätsklasse bezeichnen die Anzahl untersuchter Bäume. Zwischen den beiden Gebieten unterschied sich nur der Befall durch den Blauen Föhrenprachtkäfer (binomiales Regressionsmodell GLMM mit Wald-Chi2-Test; Blauer Föhrenprachtkäfer: Chi² $=15.951, p<0.001$; Sechszähniger Föhrenborkenkäfer: $\mathrm{Chi}^{2}=0.853, p=0.356$; Kleiner Waldgärtner: Chi $\left.^{2}=0.007, p=0.935\right)$. Die Unterschiede zwischen den Vitalitätsklassen waren unabhängig vom Gebiet (keine signifikante Interaktion) für alle drei Arten signifikant (Blauer Föhrenprachtkäfer: Chi $^{2}=47.500, p<0.001$; Sechszähniger Föhrenborkenkäfer: Chi² $=12.256, p=0.031$; Kleiner Waldgärtner: $\mathrm{Chi}^{2}=2.912, p<0.001$ )

Der Sechszähnige Föhrenborkenkäfer war nur in geringen Dichten vorhanden und wurde deshalb in Abbildung 6 nicht berücksichtigt. Eine gleichzeitige Besiedlung durch den Föhrenstangenrüssler und den Sechszähnigen Föhrenborkenkäfer wurde in keinem der ausgebrüteten Stämme festgestellt. Die beiden Arten scheinen sich gegenseitig auszuschliessen.

Die Holzwespe Sirex noctilio entwickelt sich in Europa im Holz absterbender oder frisch abgestorbener Föhren. Sie wurde von hier in andere Kontinente verschleppt und verursacht dort in Föhrenplantagen wegen ihres symbiotischen Pilzes und des toxischen Schleims grosse ökonomische Schäden (Slippers et al 2012). In Europa ist sie aber forstwirtschaftlich irrelevant und befiel auch in dieser Untersuchung im Wallis nur Bäume mit mehr als 50\% Nadelverlust (Wermelinger et al 2008, Wermelinger \& Thomson 2012).

\section{Föhrenmortalität 2016/2017}

Im Herbst 2016 machte sich im Raum Visp und Brig erneut eine starke Mortalität unter den Waldföhren bemerkbar. Dies war der Anlass für eine weitere Untersuchung. Am Nordosthang eingangs des Vispertals und am Nordhang oberhalb Brig-Glis wurden im Sommer 2017 insgesamt 104 Föhren gefällt. Ihre Kronen wurden anhand des Anteils grüner Triebe in der Krone in fünf Vitalitätsklassen eingeteilt: $0=$ tot (ganze Krone braun), $1=1-25 \%$ grüne Triebe, $2=26-50 \%$ grüne Triebe, $3=51-75 \%$ grüne Triebe, $4=76-99 \%$ grüne Triebe, $5=$ ganze Krone grün. Von jedem Baum wurde je ein Stammstück von $20 \mathrm{~cm}$ Länge auf Brusthöhe und am Kronenansatz sowie ein Aststück der Krone von $30 \mathrm{~cm}$ Länge herausgeschnitten. Diese Probestücke wurden sorgfältig geschält und quantitativ auf Spuren von Insektenbefall untersucht. Dabei wurden die Frassspuren des Sechszähnigen Föhrenborkenkäfers, des Zwölfzähnigen Föhrenborkenkäfers (Ips sexdentatus), des Kleinen und des Grossen Waldgärtners (Tomicus piniperda) sowie des Blauen Föhrenprachtkäfers unterschieden.

Beim Vergleich der beiden Standorte fällt auf, dass der Anteil befallener Bäume in Visp viel höher war als in Brig, allerdings war der Unterschied nur für den Blauen Föhrenprachtkäfer signifikant (Abbildung 7). An beiden Standorten waren der Blaue Föhrenprachtkäfer, der Sechszähnige Föhrenborkenkäfer und der Kleine Waldgärtner am häufigsten. Die Befallsraten dieser Arten zeigten klare Unterschiede zwischen den Vitalitätsklassen. Am eindrücklichsten war der Befall durch den Blauen Föhrenprachtkäfer in Visp: Während Bäume mit völlig grüner Krone (Vitalitätsklasse 5) unbesiedelt blieben, zeigten schon solche mit maximal einem Viertel toter Triebe (Vitalitätsklasse 4) Befall durch diesen Käfer. Dabei waren die Larven häufig noch in einem sehr jungen Stadium, die Besiedlung konnte also erst kurz zuvor (im Frühling) stattgefunden haben. Dies zeigt, dass der Befall nicht die Ursache des Vitalitätsverlusts der Bäume war, sondern dessen Folge. Die Befallsrate des Blauen Föhrenprachtkäfers stieg mit abnehmender Vitalität kontinuierlich an und erreichte bei den abgestorbenen Bäumen fast 100\%. Der Sechszähnige Föhrenborkenkäfer besiedelte die Bäume weniger häufig und nur solche mit weniger als 50\% grünen Trieben (Klassen 0-2).

In Brig blieben 63\% der Bäume ohne jeglichen Käferbefall (Ausnahme: der phytosanitär bedeutungslose Weiche Nagekäfer), darunter alle mit mindestens 25\% lebenden Trieben (Klassen 2-5). Die Hälfte der toten Bäume wies Befall durch den Blauen Föhrenprachtkäfer auf. Der Kleine Waldgärtner, dessen Triebfrass sehr auffällig werden kann, spielte offensichtlich beim Absterbeprozess der Föhren keine Rolle. Die Tatsache, dass in Brig die Hälfte aller toten Bäume keinen Insektenbefall hatte, zeigt, dass viele Bäume aus anderen Gründen abstarben. 


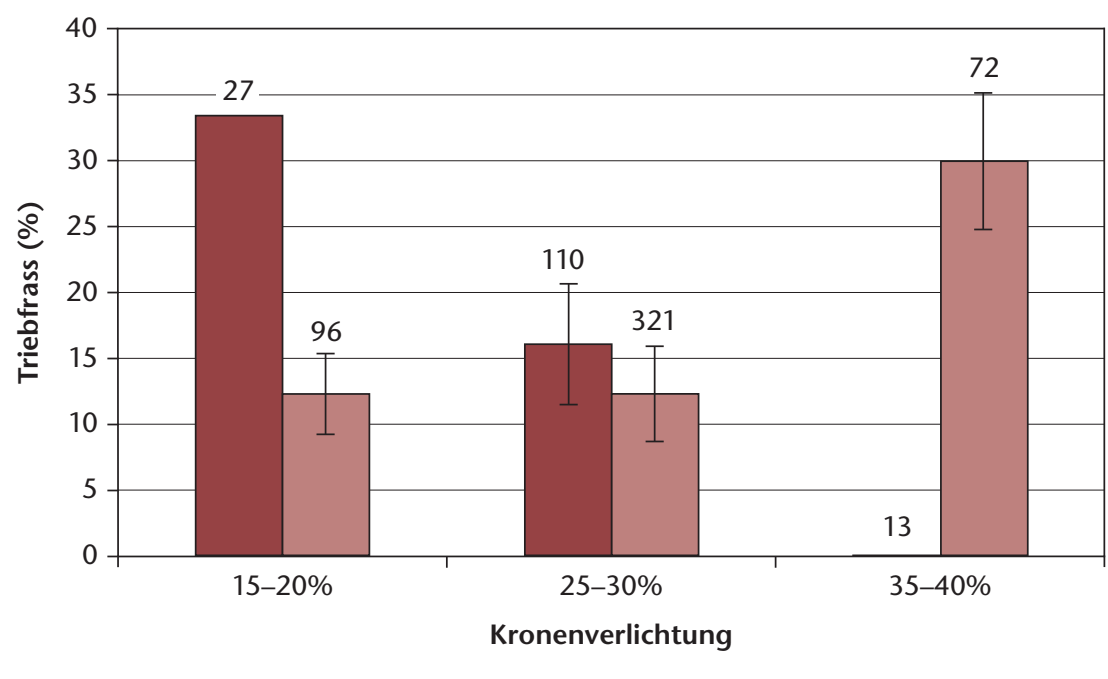

Abb 8 Prozentsatz der vom Waldgärtner (Tomicus spp.) im Jahr 2015 für den Reifungsfrass befallenen Triebe im Pfynwald in Abhängigkeit von der festgestellten Kronenverlichtung. Die Zahlen oberhalb der Säulen bezeichnen die Anzahl untersuchter Triebe (binomiales Regressionsmodell GLMM mit Wald-Chi2-Test; Kronenverlichtung: Chi $^{2}=6.744$, $p=0.034$; Bewässerung: Chi $=0.822, p=0.663$; Kronenverlichtung $\times$ Bewässerung: $\left.\mathrm{Chi}^{2}=15.428, p=0.001\right)$.

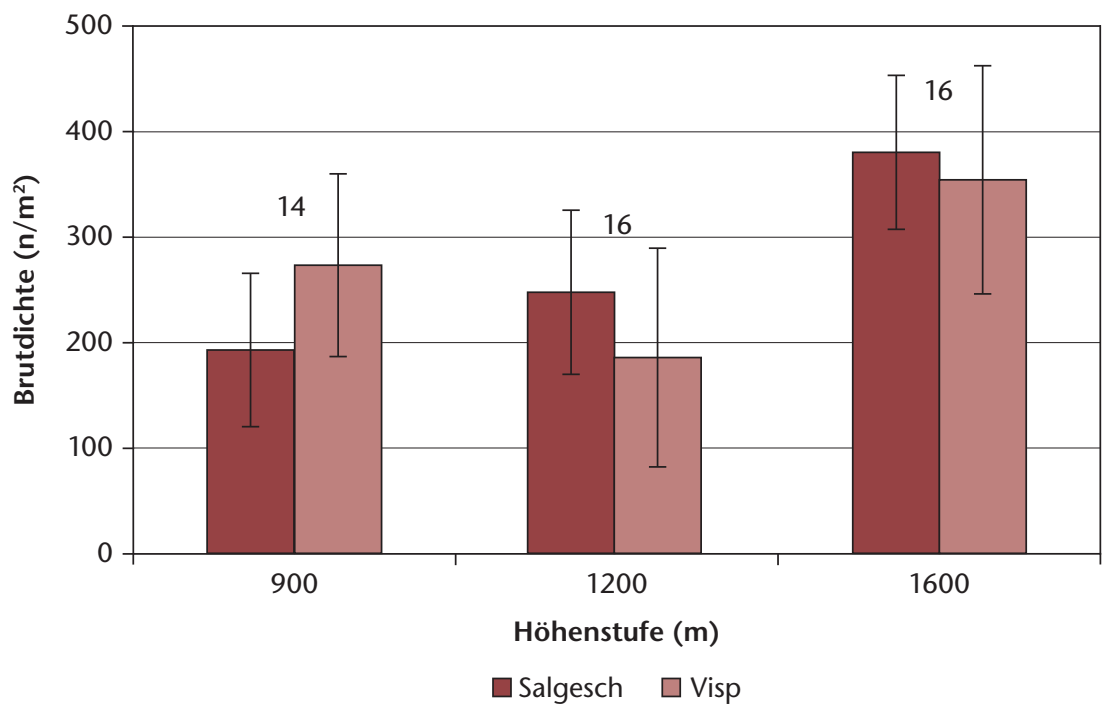

Abb 9 Bruterfolg des Sechszähnigen Föhrenborkenkäfers (Ips acuminatus), des Waldgärtners (Tomicus spp.) und des Blauen Föhrenprachtkäfers (Phaenops cyanea) in Waldföhren auf unterschiedlichen Höhenstufen in Salgesch (Südexposition) und Visp (Nordexposition). Die Zahlen oberhalb der Säulen bezeichnen die Anzahl untersuchter Bäume (Regressionsmodell $L M$, Gebiet: $F_{1,42}=0.148, p=0.702$; Höhenstufe: $F_{2,42}=2.361, p=0.107$; keine Interaktion Gebiet $\times$ Höhenstufe).

\section{Baumvitalität und Triebfrass}

Im Rahmen des Bewässerungsexperiments (Rigling et al 2018, dieses Heft) im Pfynwald wurde der Zusammenhang von Baumvitalität und Triebfrass durch die beiden Waldgärtner-Arten (Tomicus spp.) untersucht. Dazu wurden im Herbst 2015 auf den obersten Plattformen der Baumgerüste 639 Triebe aus den Kronen von 24 Waldföhren beurteilt (Abbildung 8). Im Durchschnitt waren zwischen 17 und $22 \%$ der Triebe infolge des Reifungsfrasses der adulten Käfer ausgehöhlt und verbräunt. Es zeigten sich Unterschiede zwischen den Verlichtungsklassen, die zudem abhängig vom Bewässerungsregime waren.
Während der Triebfrass in den bewässerten Flächen mit zunehmender Verlichtung zunahm, sank der Triebfrass in der Kontrollvariante mit höherer Verlichtung gegen Null. Die Käfer bevorzugen für ihren Reifungsfrass somit Bäume von hoher Vitalität, ausser wenn die Wirtspflanzen sehr gut wasserversorgt sind. Allerdings gilt diese Aussage nur für Bäume zwischen 15 und 40\% Nadelverlust, da in dieser Untersuchung solche mit einer Kronenverlichtung ausserhalb dieses Bereichs fehlten.

\section{Befallsrisiko unter Klimawandel}

Um abschätzen zu können, wie sich das Befallsrisiko durch rindenbrütende Käfer in Zukunft entwickeln könnte, wurden im Rahmen eines Interreg-Projekts Befallsdichten und die Harzabwehr von Föhren auf verschiedenen Höhenstufen erhoben. Die unterschiedlichen Höhen repräsentieren verschiedene Temperaturregimes bei sonst möglichst vergleichbaren Bedingungen. Zwischen 2009 und 2012 wurden identische Untersuchungen an je zwei Talhängen der inneralpinen Trockengebiete Churer Becken (GR), Aostatal (AO, IT) und Wallis (VS) durchgeführt. Im Herbst von jeweils zwei aufeinanderfolgenden Jahren wurden auf drei Höhenstufen (900 m, 1200 m, 1600 m ü.M.) je vier Waldföhren gefällt. Diese wurden im folgenden Frühling von den ausschwärmenden Insekten besiedelt. Im Juni wurden pro Baum je zwei Stamm- (auf Brusthöhe und am Kronenansatz) und Aststücke von $80 \mathrm{~cm}$ Länge herausgeschnitten und in Fotoeklektoren über zwei Jahre ausgebrütet (Abbildung 3). Die geschlüpften Insekten wurden anschliessend bis zur Art bestimmt. Zusätzlich wurden lebende Föhren bezüglich Harzfluss und Holzanatomie untersucht. Hier werden die Resultate aus den Walliser Versuchsorten Salgesch und Visp vorgestellt.

Am südexponierten Hang bei Salgesch zeigte sich eine mit der Höhe kontinuierlich zunehmende Besiedlungsdichte der drei aggressiven Käfer Sechzähniger Föhrenborkenkäfer, Kleiner Waldgärtner und Blauer Föhrenprachtkäfer (Abbildung 9). Am nordexponierten Hang bei Visp war der Verlauf weniger klar, da die mittlere Stufe tendenziell die geringste Besiedlungsdichte aufwies. Werden hingegen die Daten aus allen drei Gebieten (VS, GR, AO) kombiniert, nehmen die Dichten der aggressiven Käferarten mit der Höhe signifikant zu, allerdings gibt es auch eine Interaktion mit der Exposition (nicht dargestellt). Die zahlenmässig dominanten Arten waren die beiden Waldgärtner-Arten, von denen im Wallis bis zu 900 Stück pro Quadratmeter Rinde ausschlüpften. Es ist aber zu beachten, dass die Besiedlung an gefällten Bäumen stattfand.

Der Befund, dass in tieferen Lagen mit höheren Temperaturen weniger aggressive Rindenbrüter 
Abb 10 Tangentialschnitt durch das äussere Xylem einer Waldföhre. Sichtbar sind die axialen Tracheiden (rot) und die radialen Markstrahlen (blau/weiss). In der Bildmitte befindet sich ein mehrreihiger Markstrahl mit einem Harzkanal.

Foto: Barbara Kinigadner

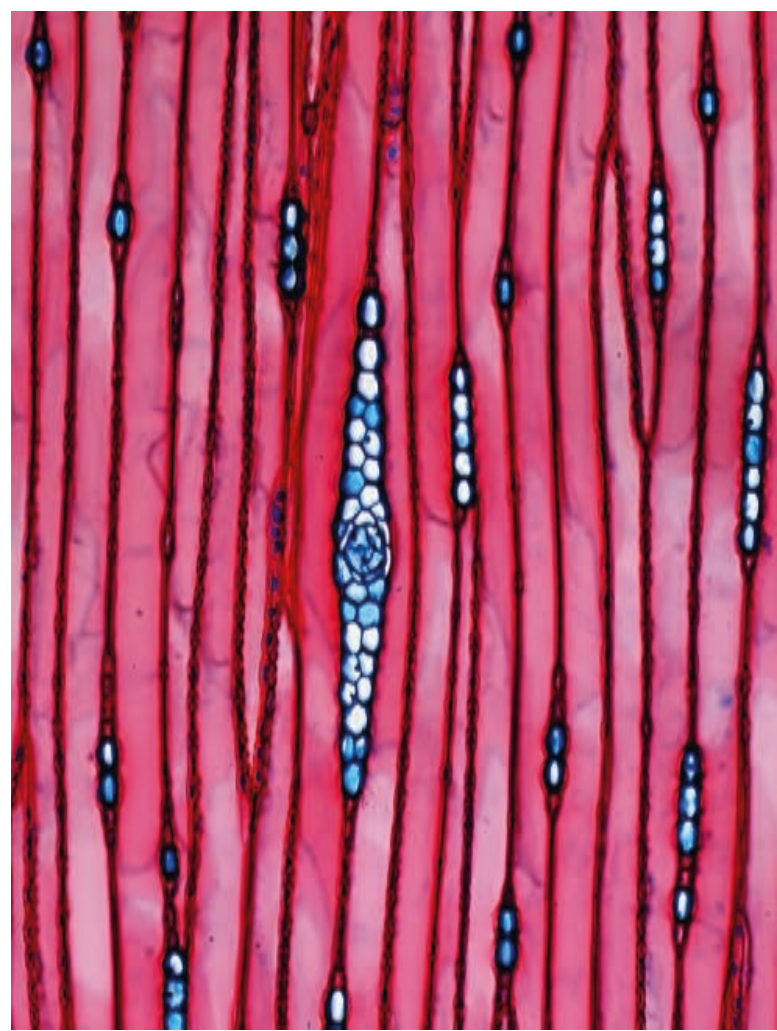

aus den Bruthölzern ausschlüpften, mag erstaunen. Einerseits nahm aber die Dichte an natürlichen Feinden, v.a. Schlupfwespen, mit den höheren Temperaturen tiefer Lagen zu und damit auch die resultierende Mortalität der rindenfressenden Insekten. Andererseits könnte diese Höhenverteilung auch auf den in tieferen Lagen stärkeren Harzfluss und damit eine höhere Abwehrfähigkeit der Bäume zurückzuführen sein. Messungen zeigten, dass der Harzfluss mit der Höhe signifikant abnahm (LM, Gebiet: $\mathrm{F}_{1,93}=$ 0.181, $\mathrm{p}=0.671$; Höhenstufe: $\mathrm{F}_{2,93}=3.967, \mathrm{p}=0.022$ ). Holzanatomische Analysen (Abbildung 10) ergaben keine Unterschiede in der Dichte der Harzkanäle auf verschiedenen Höhenstufen, was bedeutet, dass der höhere Harzfluss in tiefen Lagen rein temperaturbedingt auf die geringere Viskosität zurückzuführen sein dürfte.

\section{Diskussion und Schlussfolgerungen}

Geschwächte und absterbende Bäume sind häufig von rindenbrütenden Insekten besiedelt. Dabei stellt sich die Frage, ob die Insekten der Grund für die Schwächung oder das Absterben eines Baumes sind oder ob der Insektenbefall umgekehrt die Folge des Vitalitätsverlusts eines Baumes ist. Unsere in den vergangenen 15 Jahren im Wallis an Waldföhren durchgeführten Untersuchungen zeigen, dass keine allgemein gültigen Muster festzustellen sind. Es gab Situationen, in denen die Bäume erst bei starker Schwächung besiedelt wurden oder sogar ohne jeglichen Insektenbefall abstarben. In anderen Situ- ationen befielen bestimmte der drei aggressiven Käferarten die Bäume schon bei geringem Vitalitätsverlust und verursachten ihr Absterben. Völlig vitale Föhren wurden jedoch kaum von Schädlingen besiedelt, was den Schluss nahelegt, dass Insekten im untersuchten System nur in Interaktion mit anderen Faktoren zum Absterben von Bäumen führten.

$\mathrm{Zu}$ den potenziellen Schadinsekten gehören in erster Linie der Sechszähnige Föhrenborkenkäfer und der Blaue Föhrenprachtkäfer, während der Kleine Waldgärtner von geringer Bedeutung ist. Die erstgenannten Arten vermögen zwar keine vitalen Bäume mit voll belaubter, grüner Krone zu besiedeln, aber bereits eine mässige und möglicherweise auch vorübergehende Schwächung mit 20 bis 30\% Nadelverlust setzt die Vitalität der Bäume soweit herab, dass sie für diese beiden Käferarten attraktiv und besiedlungstauglich werden. Dies führt in der Folge meist zum Absterben der Bäume. Auslöser für eine solche Schwächung ist meistens Trockenheit. Die Käfer können auf Jahre mit mangelnden Frühjahrsniederschlägen erstaunlich schnell mit erhöhtem Befall reagieren. Dabei gab es in unseren Untersuchungen aber immer auch einen gewissen Anteil von Bäumen, die ohne Insektenbefall abstarben. Dies könnten möglicherweise Bäume sein, die eine sehr langsame Schwächung mit gradueller Abnahme der Phloemqualität erfuhren. Diese Hypothese könnte mit den Zuwachsraten aus Jahrringanalysen getestet werden. Wenn solche Bäume für die erwähnten aggressiven Käferarten nie attraktiv werden, werden sie erst später von Totholzinsekten besiedelt. Im Gegensatz dazu haben Wirtsbäume, die einen plötzlichen Stress erleiden, ein intaktes und qualitativ hochwertiges Phloem, aber bereits eine geringe Abwehrkraft.

Beide Phänomene - kein Käferbefall von vitalen Bäumen und das Absterben von Bäumen ohne jegliche Insekten - zeigen, dass Borken- und Prachtkäfer auch bei längerer Trockenheit nicht die Verursacher des verbreiteten Absterbens von Waldföhren sind, wie dies zum Beispiel bei einer Massenvermehrung des Buchdruckers (I. typographus) bei Fichte (Picea abies) der Fall sein kann. Insbesondere der Blaue Föhrenprachtkäfer kann jedoch schon bei leichtem Vitalitätsschwund Bäume besiedeln, schwächen oder zum Absterben bringen. Die Tatsache, dass die in der Vitalitätsklasse 4 (76-99\% grüne Triebe) gefundenen Prachtkäfer als frisch geschlüpfte Junglarven vorlagen, zeigt, dass nicht die Larven der Grund für den geringen Vitalitätsverlust waren, sondern dass die Bäume bereits bei der Eiablage durch die Mutterkäfer geschwächt waren.

Während dieses schwächebedingten Absterbeprozesses stellt sich nach dem Befall der aggressiven Käferarten auch eine Sukzession von verschiedenen Begleitarten von Borken-, Rüssel- und Bockkäfern ein, die das noch lebende Phloem und das noch 
feuchte Holz als Nahrungssubstrat nutzen. Neben Insekten können zudem Pilze oder Misteln eine wichtige Rolle im Absterbeprozess spielen (Dobbertin \& Rigling 2006, Wermelinger et al 2006). Eine gemeinsame Analyse von Insekten-, Pilz-, Nematoden- und Jahrringdaten wird weitere Aufschlüsse geben.

Was allerdings der fortschreitende Klimawandel für das Befallsrisiko durch Schadinsekten bedeutet, ist schwierig zu beurteilen. So fliesst das Baumharz bei höheren Temperaturen zwar rascher, was die Abwehr von Käfern begünstigen würde, aber die zunehmende Trockenheit dürfte die Harzproduktion einschränken. Auch fördern höhere Temperaturen die Entwicklungsgeschwindigkeit sowohl der potenziellen Schadinsekten als auch ihrer natürlichen Gegenspieler. Unsere Untersuchungen entlang der Höhengradienten zeigen einen geringeren Bruterfolg von aggressiven Käfern bei höheren Temperaturen, was wahrscheinlich auf eine effektivere Kontrolle durch Antagonisten zurückzuführen ist. Wie die komplexen Wechselwirkungen zwischen Wirtsbäumen, Rindenbrütern und natürlichen Feinden sich unter verändertem Klima tatsächlich entwickeln werden, ist aber kaum abschätzbar. Aufgrund der bisherigen Ereignisse ist jedoch davon auszugehen, dass es in den inneralpinen Trockentälern vor allem in den tieferen Lagen zu weiteren lokalen oder regionalen Mortalitätswellen bei Föhren kommen wird und dass Käferbefall eine begleitende bis tragende Rolle spielen wird.

Eingereicht: 24. November 2017, akzeptiert (mit Review): 29. Juni 2018

\section{Dank}

Wir bedanken uns ganz herzlich bei der Dienststelle für Wald, Flussbau und Landschaft des Kantons Wallis für die langjährige Unterstützung, ebenso bei den Forstbetrieben «Forst Region Leuk», «Visp und Umgebung», «Stalden und Umgebung» und «Forstrevier Burgerschaft Brig-Glis» für die tatkräftige Mithilfe von verschiedenen Mitarbeitern. Ein ebenso herzlicher Dank geht an die zahlreichen Mitarbeitenden, Kollegen, Praktikanten/innen und Lehrlinge, die in grösserem oder kleinerem Ausmass bei den langjährigen Untersuchungen mithalfen: D. Bösch, B. Fecker†, M. Furrer†, A. Gall, H. Gärtner, M. Gees, B. Kinigadner, K. Kleeb, Q. Kupper, R. Lazaro Martin, S. Mathis, D. Nievergelt, M. Schmidheiny, D. Steiner, A. Streit, O. Zuberbühler.

\section{Literatur}

ALLEN CD, MACALADY AK, CHENCHOUNI H, BACHELET D, MCDOWELL N ET AL (2010) A global overview of drought and heat-induced tree mortality reveals emerging climate change risks for forests. For Ecol Manage 259: 660-684.
DAS AJ, STEPHENSON NL, DAVIS KP (2016) Why do trees die? Characterizing the drivers of background tree mortality. Ecology 97: 2616-2627.

DOBBERTIN M, RIGLING A (2006) Pine mistletoe (Viscum album ssp. austriacum) contributes to Scots pine (Pinus sylvestris) mortality in the Rhone valley of Switzerland. For Pathol 36: 309-322.

ECKELT A, MÜLLER J, BENSE U, BRUSTEL H, BUSSLER H ET AL (2017) "Primeval forest relict beetles" of Central Europe: a set of 168 umbrella species for the protection of primeval forest remnants. J Ins Conserv 22: 15-28.

GAYLORD ML, KOLB TE, POCKMAN WT, PLAUT JA, YEPEZ EA ET AL (2013) Drought predisposes piñon-juniper woodlands to insect attacks and mortality. New Phytol 198: 567-578.

KARBAN R, BALDWIN IT (1997) Induced responses to herbivory. Chicago: Univ Chicago Press. 319 p.

KROKENE P (2015) Conifer defense and resistance to bark beetles. In: Vega FE, Hofstetter RW, editors. Bark beetles: Biology and ecology of native and invasive species. London: Academic Press. pp. 177-207.

LARSSON S (2002) Resistance in trees to insects - an overview of mechanisms and interactions. In: Wagner MR, Clancy KM, Lieutier F, Paine TD, editors. Mechanisms and deployment of resistance in trees to insects. Dordrecht: Kluwer Academic Publishers. pp. 1-29.

MONNERAT C, BARBALAT S, LACHAT T, GONSETH Y (2016) Rote Liste der Prachtkäfer, Bockkäfer, Rosenkäfer und Schröter. Gefährdete Arten der Schweiz. Bern: Bundesamt Umwelt. $118 \mathrm{p}$.

MÜLLER J, BUSSLER H, GOSSNER M, RETTELBACH T, DUELLI P (2008) The European spruce bark beetle Ips typographus in a national park: from pest to keystone species. Biodivers Conserv 17: 2979-3001.

NETHERER S, MATTHEWS B, KATZENSTEINER K, BLACKWELL E, HENSCHKE P ET AL (2015) Do water-limiting conditions predispose Norway spruce to bark beetle attack? New Phytol 205: 1128-1141.

PHILLIPS MA, CROTEAU RB (1999) Resin-based defenses in conifers. Trends Plant Sci 4: 184-190.

RAFFA KF, AUKEMA BH, BENTZ BJ, CARROLL AL, HICKE JA ET AL (2008) Cross-scale drivers of natural disturbances prone to anthropogenic amplification: The dynamics of bark beetle eruptions. BioScience 58: 501-517.

REBETEZ M, DOBBERTIN M (2004) Climate change may already threaten Scots pine stands in the Swiss Alps. Theor Appl Climatol 79: 1-9.

RIGLING A, MOSER B, FEICHTINGER L, GÄRTNER H, GIUGGIOLA A ET AL (2018) 20 Jahre Waldföhrensterben im Wallis: Rückblick und aktuelle Resultate. Schweiz Z Forstwes 169: 242-250. doi: 10.3188/szf.2018.0242

SLIPPERS B, DE GROOT P, WINGFIELD MJ, EDITORS (2012) The Sirex woodwasp and its fungal symbiont. Research and management of a worldwide invasive pest. Dordrecht: Springer. $301 \mathrm{p}$.

WERMELINGER B, SEIFERT M (1999) Temperature-dependent reproduction of the spruce bark beetle Ips typographus, and analysis of the potential population growth. Ecol Entomol 24: 103-110.

WERMELINGER B, POLOMSKI J, HEINIGER U, RIGLING D, RIGLING A (2006) Föhrensterben im Wallis: Welche Rolle spielen Schädlinge und Krankheiten? Wald Holz 87 (12): 58-61.

WERMELINGER B, RIGLING A, SCHNEIDER MATHIS D, DOBBERTIN M (2008) Assessing the role of bark- and wood-boring insects in the decline of Scots pine (Pinus sylvestris) in the Swiss Rhone valley. Ecol Entomol 33: 239-249.

WERMELINGER B, THOMSON IM (2012) The woodwasp Sirex noctilio and its associated fungus Amylostereum areolatum in 
Europe. In: Slippers B, de Groot P, Wingfield MJ, editors. The Sirex woodwasp and its fungal symbiont. Research and management of a worldwide invasive pest. Dordrecht: Springer. pp. 65-80.

WERMELINGER B (2017) Insekten im Wald - Vielfalt, Funktionen und Bedeutung: Bern: Haupt. 367 p.
WERMELINGER B, JAKOBY O (IM DRUCK) Borkenkäfer. In: Wohlgemuth T, Seidl R, Jentsch A, editors. Störungsökologie: Revolution in Pflanzengemeinschaften. Bern: Haupt.

WILLIAMS AP, ALLEN CD, MACALADY AK, GRIFFIN D, WOODHOUSE CA ET AL (2013) Temperature as a potent driver of regional forest drought stress and tree mortality. Nat Clim Chang 3: 292-297.

\section{Influence du climat et de la vitalité des arbres sur l'infestation des pins sylvestres par les insectes corticoles}

Même si le développement des insectes dépend avant tout de la température, les composants des plantes-hôtes n'en jouent pas moins un rôle déterminant. En effet, leur qualité nutritionnelle, en particulier leur teneur en azote, est très importante pour la pullulation des insectes, tandis que les substances de défense toxiques des plantes influencent la colonisation et le développement des insectes. Or en cas de sécheresse, la production de ces substances de défense est souvent fortement limitée. En Valais, différentes études qui portent sur l'influence de la température et de la sécheresse sur les insectes corticoles ont été menées ces 15 dernières années. Elles ont mis en évidence les espèces au plus fort potentiel d'infestation: le scolyte acuminé (Ips acuminatus), le bupreste bleu du pin (Phaenops cyanea) et - dans une moindre mesure - l'hylésine mineur (Tomicus minor). Ces derniers ont également réussi à coloniser les pins sylvestres de vitalité relativement élevée. De façon générale, l'infestation par ces espèces et par d'autres scolytes corticoles augmentait avec une défoliation croissante du houppier. Les insectes prédateurs présentaient le même schéma de colonisation. L'intensité des forages de maturation de l'hylésine dépendait de la défoliation du houppier, tout en étant très influencée par l'approvisionnement en eau des arbres. Pour les années étudiées, l'évolution dans le temps de la densité d'infestation des insectes corticoles agressifs correspondait assez clairement à un indice de stress hydrique des arbres-hôtes. Mais les taux d'infestation comportaient souvent de nettes différences régionales. Les densités de colonisation augmentaient avec l'altitude, ce qui est probablement lié au nombre décroissant de leurs ennemis naturels et au flux de résine réduit en présence de températures inférieures.

\section{Effects of climate and tree vitality on the infestation of Scots pines by bark-dwelling insects}

The development of insects depends not only on temperature but also on the chemical composition of their host plant. On the one hand, the nutritional quality and in particular the nitrogen content affects insect propagation, and on the other hand, toxic defense compounds impact the colonization by and the survival of insects. The production of such plant defense compounds is restricted under dry conditions. In the Valais, Switzerland, studies on the influence of temperature and drought on bark-dwelling insects were carried out during the last 15 years. The buprestid Phaenops cyanea, the bark beetle Ips acuminatus, and to a lesser extent the bark beetle Tomicus minor showed the greatest potential for damage. They were able to colonize Scots pines within a large range of vitality levels, measured as crown needle loss, but with large regional differences. Infestation levels by these and other bark-dwelling beetles increased with increasing needle loss. The same pattern was found for predatory insects. The intensity of maturation feeding of Tomicus bark beetles depended on crown transparency interacting with tree water supply. The temporal development of infestations by aggressive bark-dwelling insects followed the course of an index of tree drought stress quite closely. In general, infestation levels showed large regional differences. Breeding density of aggressive bark-dwelling insects increased with elevation which can be attributed to decreasing numbers of natural enemies and to reduced resin flow at lower temperatures. 